\title{
ER Platforms for Membrane Lipid Dynamics
}

The endoplasmic reticulum (ER) is the foundation of the eukaryotic cell. It forms the nuclear envelope and is in contact with other membrane-bound organelles regarded as hallmarks of eukaryotic cells. The ER is also a major site for cellular metabolism, including both anabolic and catabolic processes. In particular, it is well recognized for its roles in protein biogenesis, quality control, and secretion. However, and generally less understood, the ER is also a key site for the synthesis of lipids that are supplied to the various membrane compartments in the cell. Accordingly, the ER forms a vast membrane network throughout the cell, and it is now becoming evident that the ER is organized into specialized sub-domains that execute discrete functions in membrane lipid metabolism and transport. As such, studies on ER architecture and function have been integral to cell biology research. To highlight scientific advances and the building excitement in this field, we have put together a collection of sixteen timely review articles. This series, entitled "ER Platforms for Membrane Dynamics", presents the latest developments in our understanding of vital roles for distinct ER domains in membrane lipid metabolism and trafficking. Themes include membrane lipid synthesis and remodelling, transport mechanisms from the ER, lipid droplet biogenesis, the ER in autophagy, and ER membrane lipid sensing and homeostasis.

This special issue aims to stimulate discussions in ER membrane lipid biology and to feature under-appreciated facets of the ER in cell biology. The first four reviews cover specific topics involving regulation of membrane lipid metabolism and remodeling in the ER. Kwiatek, Han, and Carman discuss regulation of phosphatidic acid and diacylglycerol in the ER and how their metabolic flux controls the synthesis of phospholipids and the neutral storage lipid triacylglycerol. In a related topic, Lee and Ridgway elucidate how metabolic channeling in the ER regulates the balance between phospholipid and storage lipid synthesis pathways in response to nutritional status and growth factors. In addition to de novo lipid synthesis, the ER is also an important site for phospholipid acyl chain remodeling. Patton-Vogt and de Kroon summarize new insights into the machinery for phospholipid degradation and remodeling in the ER and their roles in membrane bilayer organization. In addition to glyceroand phospholipids, de novo sphingolipid biosynthesis is initiated in the ER. Zelnik, Ventura, Kim, Silva, and Futerman focus on the precise regulation of ceramide sphingolipid species and their influence on the integrity and biophysical properties of biological membranes.

Next, the theme of the series expands to encompass mechanisms for coupling lipid synthesis and non-vesicular transport. Blunsom and Cockcroft summarize rate-limiting steps for phosphatidylinositol synthesis including transfer of phosphatidic acid to the ER during the phosphoinositide cycle. Related to this subject, Balla, Sengupta, and Kim highlight how transfer of phosphatidylinositol out of the ER serves to promote its synthesis. Nishimura and Stefan extend upon this topic and discuss additional reciprocal relationships between lipid metabolism and non-vesicular transport. Jeyasimman and Saheki focus on the synaptotogamin-like mitochondrial-lipid-binding domain protein family and their roles in transferring a variety of lipids between the ER and other organelles. Two review articles specifically cover non-vesicular lipid exchange between the ER and membrane compartments in the endo-lysosomal system. Di Mattia, Tomasetto, and Alpy describe the protein complexes that form and function at ER-endosomal membrane contact sites. Lee and Blackstone present roles for these organelle contacts in sterol lipid exchange, endolysosomal membrane dynamics, and calcium signaling.

The ER is the origin of vesicular carriers in the secretory pathway and it serves in the biogenesis of certain membrane-bound organelles. Funato, Riezman, and Muniz provide an extensive discussion of how membrane lipid synthesis in the ER supports both non-vesicular and vesicular transport to organelles along the secretory pathway. In addition to secretory trafficking, the ER is involved in the formation of autophagosomes that are targeted to lysosomes. Ktistakis discusses evidence implicating specialized ER domains as a membrane lipid source necessary for de novo biogenesis of the isolation membrane during autophagosome formation. In addition to vesicular intermediates, the ER is the birthplace of lipid droplets that store neutral lipids including triacylglycerol and sterol esters that provide energy sources upon starvation conditions. Two review articles in this series describe roles for specialized regions and factors in the ER during lipid droplet biogenesis. Henne, 
Goodman, and Hariri describe the lipid synthesis enzymes and structural components involved in de novo lipid droplet formation and their distinct roles in cellular homeostasis upon various stress conditions. Nettebrock and Bohnert also discuss proteins required for lipid droplet formation and further highlight how ER membrane lipids cooperate in turn with these protein factors to promote and specify lipid droplet biogenesis and dynamics as needed in response to cellular status.

Disruptions in membrane lipid synthesis impair ER function and induce ER stress responses. Likewise, the improper accumulation of lipids due to impaired transport also causes ER stress. The final two reviews in this special issue focus on how cells deal with ER stress caused by membrane lipid imbalances. Goder, Alanis-Dominquez, and BustamanteSequeirios describe intricate regulatory mechanisms for cross talk between membrane lipid metabolism and proteostasis via the ER-associated protein degradation pathway. Covering another aspect of ER stress responses, Fun and Thibault discuss emerging concepts involving how distinct branches of the unfolded protein response pathway may be differentially induced and respond to membrane bilayer stress versus protein misfolding in the ER. 